\title{
AFFINE SURFACES WHOSE GEODESICS ARE PLANAR CURVES
}

\author{
LUC VRANCKEN
}

(Communicated by Christopher Croke)

\begin{abstract}
We study the geometry of nondegenerate affine surfaces $M^{2}$ in $\mathbb{R}^{4}$, with respect to the Burstin-Mayer, the Weise-Klingenberg and the equiaffine transversal plane bundle. A classification is obtained of the surfaces whose geodesics with respect to the induced connection are planar curves.
\end{abstract}

\section{INTRODUCTION}

In this paper, we study nondegenerate affine surfaces $M^{2}$ in $\mathbb{R}^{4}$. For such surfaces there exist infinitely many canonically determined (and in general different) affine transversal planes (see [SV]). Among this family there are three which have been closely studied in the literature, namely the Burstin-Mayer transversal plane bundle ([BM]), the Weise-Klingenberg transversal plane bundle $([\mathrm{K}])$ and the equiaffine transversal plane bundle, which was introduced in [NV]. For more details and a geometric motivation of this last transversal bundle we refer to [NV] and [DMVV]. Here, we prove the following theorem.

Main Theorem. Let $M^{2}$ be a nondegenerate affine surface in $\mathbb{R}^{4}$. Denote by $\nabla^{1}$ (resp. $\nabla^{2}$ and $\nabla^{3}$ ) the connection induced by the equiaffine transversal plane bundle (resp. the Burstin-Mayer transversal plane bundle, the Weise-Klingenberg transversal plane bundle). Then the following conditions are equivalent:

(1) The $\nabla^{1}$-geodesics of $M$ are planar curves.

(2) The $\nabla^{2}$-geodesics of $M$ are planar curves.

(3) The $\nabla^{3}$-geodesics of $M$ are planar curves.

(4) $M$ is affine equivalent with either one of

$$
x(u, v)=\left(u, v, \frac{1}{2}\left(u^{2}-v^{2}\right), u v\right) \text { and } x(u, v)=\left(u, u^{2}, v, v^{2}\right) \text {. }
$$

\section{THE AFFINE METRIC AND THE DIFFERENT TRANSVERSAL PLANE BUNDLES}

Here, we describe how to obtain the different transversal plane bundles. For details, we refer to [NV] and [SV]. Consider $\mathbb{R}^{4}$ with its standard affine con-

Received by the editors November 23, 1993 and, in revised form, May 31, 1994.

1991 Mathematics Subject Classification. Primary 53A15; Secondary 53B25.

Key words and phrases. Affine differential geometry, higher codimension, planar geodesics.

The author is a Senior Research Assistant of the National Fund for Scientific Research (Belgium). 
nection $D$ and its parallel volumeform $\omega$ given by the determinant. Let $M^{2}$ be a surface in $\mathbb{R}^{4}$ and $\sigma$ a transversal plane bundle. We denote by $\xi_{1}$ and $\xi_{2}$ two local vector fields which form a local basis of $\sigma$. Then for tangent vector fields $X$ and $Y$ to $M$, we have the following unique decompositions:

$$
\begin{aligned}
& D_{X} Y=\nabla_{X} Y+h^{1}(X, Y) \xi_{1}+h^{2}(X, Y) \xi_{2}, \\
& D_{X} \xi_{1}=-S_{1} X+\tau_{1}^{1}(X) \xi_{1}+\tau_{1}^{2}(X) \xi_{2}, \\
& D_{X} \xi_{2}=-S_{2} X+\tau_{2}^{1}(X) \xi_{1}+\tau_{2}^{2}(X) \xi_{2} .
\end{aligned}
$$

We call $\nabla$ the affine connection induced by the transversal plane bundle $\sigma$. The cubic forms $C^{1}$ and $C^{2}$ are defined by

$$
\begin{aligned}
& C^{1}(X, Y, Z)=\left(\nabla_{X} h^{1}\right)(Y, Z)+\tau_{1}^{1}(X) h^{1}(Y, Z)+\tau_{2}^{1}(X) h^{2}(Y, Z), \\
& C^{2}(X, Y, Z)=\left(\nabla_{X} h^{2}\right)(Y, Z)+\tau_{1}^{2}(X) h^{1}(Y, Z)+\tau_{2}^{2}(X) h^{2}(Y, Z) .
\end{aligned}
$$

Then, if $M$ is nondegenerate, we can use $D$ and $\omega$ to define a metric $g$ on $M$ (see [NV]). If $g$ is definite, if necessary by interchanging two coordinates in $\mathbb{R}^{4}$, we may assume $g$ is positive definite.

Let $u=\left\{X_{1}, X_{2}\right\}$ be an orthonormal frame, i.e., $g\left(X_{i}, X_{j}\right)=(\varepsilon)^{i} \delta_{i j}$, for $i, j \in\{1,2\}$, where $\varepsilon=1$ if $g$ is positive-definite and $\varepsilon=-1$ if $g$ is indefinite. Then, in any transversal plane bundle $\sigma$, there exists a unique local basis $\left\{\xi_{1}, \xi_{2}\right\}$ of $\sigma$ such that

$$
\begin{array}{lll}
\omega\left(X_{1}, X_{2}, \xi_{1}, \xi_{2}\right)=1, & & \\
h^{1}\left(X_{1}, X_{1}\right)=1, & h^{1}\left(X_{2}, X_{2}\right)=-\varepsilon, & h^{1}\left(X_{1}, X_{2}\right)=0, \\
h^{2}\left(X_{1}, X_{1}\right)=0, & h^{2}\left(X_{2}, X_{2}\right)=0, & h^{2}\left(X_{1}, X_{2}\right)=1 .
\end{array}
$$

In order to simplify expressions, we write $C_{j k l}^{i}=C^{i}\left(X_{j}, X_{k}, X_{l}\right)$, where $i, j, k, l \in\{1,2\}$ and $u=\left\{X_{1}, X_{2}\right\}$ is a local orthonormal basis, with corresponding transversal basis $\left\{\xi_{1}, \xi_{2}\right\}$. From [NV] and [SV], we have the following characterisations of the different transversal plane bundles:

1. The equiaffine transversal plane bundle $\left(\sigma_{\mathrm{eq}}\right)$ is determined by

$$
\begin{array}{ll}
\varepsilon C_{122}^{1}-C_{111}^{1}-2 C_{211}^{2}=0, & C_{211}^{1}+\varepsilon C_{222}^{1}+\varepsilon C_{111}^{2}+C_{122}^{2}=0, \\
C_{211}^{1}-\varepsilon C_{222}^{1}+2 C_{122}^{1}=0, & C_{211}^{2}-\varepsilon C_{122}^{1}-C_{111}^{1}+\varepsilon C_{222}^{2}=0 .
\end{array}
$$

2. The Burstin-Mayer transversal plane bundle $\left(\sigma_{\mathrm{BM}}\right)$ is determined by

$$
\begin{aligned}
& 2 C_{211}^{1}+\varepsilon C_{222}^{1}+C_{122}^{2}=0, \quad \varepsilon C_{111}^{2}+C_{122}^{2}+C_{211}^{1}+\varepsilon C_{222}^{1}=0, \\
& 2 \varepsilon C_{122}^{1}+C_{111}^{1}-C_{211}^{2}=0, \quad \varepsilon C_{122}^{1}+C_{111}^{1}-\varepsilon C_{222}^{2}-C_{211}^{2}=0 .
\end{aligned}
$$

3. The Weise-Klingenberg transversal plane bundle $\left(\sigma_{\mathrm{WK}}\right)$ is determined by

$$
C_{222}^{2}=\varepsilon C_{111}^{1}, \quad C_{122}^{2}=-C_{211}^{1}, \quad C_{21 !}^{2}=\varepsilon C_{122}^{1}, \quad C_{111}^{2}=-C_{222}^{1} \text {. }
$$

From the above characterisations it follows that if there exists a transversal plane bundle $\sigma$ such that $C^{1}=C^{2}=0$, then $\sigma=\sigma_{\mathrm{eq}}=\sigma_{\mathrm{BM}}=\sigma_{\mathrm{WK}}$.

\section{Proof of the Main theorem}

Let $M^{2}$ be a nondegenerate surface in $\mathbb{R}^{4}$. We start by taking an arbitrary transversal plane bundle $\sigma$ which induces a connection $\nabla$ on the surface, and we assume that the $\nabla$-geodesics are planar curves. 
Lemma 3.1. Let $p \in M^{2}$. For every vector $v$, we have

$$
\begin{gathered}
h^{1}(v, v) C^{2}(v, v, v)-h^{2}(v, v) C^{1}(v, v, v)=0 \\
h^{1}(v, v) S_{1} v+h^{2}(v, v) S_{2} v \text { is a vector in the direction of } v
\end{gathered}
$$

Proof. Let $p \in M^{2}$ and let $\gamma$ be the $\nabla$-geodesic through $p$ in the direction of a non-zero vector $v$. Then

$$
\begin{aligned}
D_{\gamma^{\prime}} \gamma^{\prime}= & h^{1}\left(\gamma^{\prime}, \gamma^{\prime}\right) \xi_{1}+h^{2}\left(\gamma^{\prime}, \gamma^{\prime}\right) \xi_{2}, \\
D_{\gamma^{\prime}}\left(D_{\gamma^{\prime}} \gamma^{\prime}\right)= & -h^{1}\left(\gamma^{\prime}, \gamma^{\prime}\right) S_{1} \gamma^{\prime}-h^{2}\left(\gamma^{\prime}, \gamma^{\prime}\right) S_{2} \gamma^{\prime} \\
& +C^{1}\left(\gamma^{\prime}, \gamma^{\prime}, \gamma^{\prime}\right) \xi_{1}+C^{2}\left(\gamma^{\prime}, \gamma^{\prime}, \gamma^{\prime}\right) \xi_{2} .
\end{aligned}
$$

Since $\gamma^{\prime}$ is a tangent vector, the fact that $\gamma$ is planar implies that $\gamma^{\prime}, D_{\gamma^{\prime}} \gamma^{\prime}$ and $D_{\gamma^{\prime}} D_{\gamma^{\prime}} \gamma^{\prime}$ are linearly dependent. Hence

$$
h^{1}\left(\gamma^{\prime}, \gamma^{\prime}\right) C^{2}\left(\gamma^{\prime}, \gamma^{\prime}, \gamma^{\prime}\right)-h^{2}\left(\gamma^{\prime}, \gamma^{\prime}\right) C^{1}\left(\gamma^{\prime}, \gamma^{\prime}, \gamma^{\prime}\right)=0
$$

and $-h^{1}\left(\gamma^{\prime}, \gamma^{\prime}\right) S_{1} \gamma^{\prime}-h^{2}\left(\gamma^{\prime}, \gamma^{\prime}\right) S_{2} \gamma^{\prime}$ is a vector in the direction of $\gamma^{\prime}$.

We now take a local orthonormal basis $u=\left\{X_{1}, X_{2}\right\}$. We write $c=\cos t$ and $s=\sin t$ if $M^{2}$ is positive definite and $c=\cosh t$ and $s=\sinh t$ if $M^{2}$ is indefinite. Then for any vector field $X=c X_{1}+s X_{2}$, the previous lemma implies that

$$
\begin{gathered}
h^{1}(X, X) C^{2}(X, X, X)-h^{2}(X, X) C^{1}(X, X, X)=0, \\
g\left(h^{1}(X, X) S_{1} X+h^{2}(X, X) S_{2} X, X^{\perp}\right)=0,
\end{gathered}
$$

where $X^{\perp}=-\varepsilon s X_{1}+c X_{2}$. We first look at (3.2). This gives us that

$$
\begin{aligned}
0= & c^{4} g\left(S_{1} X_{1}, X_{2}\right)+s^{4} g\left(S_{1} X_{2}, X_{1}\right) \\
& +c^{3} s\left(-\varepsilon g\left(S_{1} X_{1}, X_{1}\right)+g\left(S_{1} X_{2}, X_{2}\right)+2 g\left(S_{2} X_{1}, X_{2}\right)\right) \\
& +c^{2} s^{2}\left(-\varepsilon g\left(S_{1} X_{2}, X_{1}\right)-\varepsilon g\left(S_{1} X_{1}, X_{2}\right)-2 \varepsilon g\left(S_{2} X_{1}, X_{1}\right)+2 g\left(S_{2} X_{2}, X_{2}\right)\right. \\
& +c s^{3}\left(g\left(S_{1} X_{1}, X_{1}\right)-\varepsilon g\left(S_{1} X_{2}, X_{2}\right)-2 \varepsilon g\left(S_{2} X_{2}, X_{1}\right) .\right.
\end{aligned}
$$

Since the above equation has to be valid for all values of $t$, we deduce that

$$
\begin{aligned}
& g\left(S_{1} X_{1}, X_{2}\right)=0, \quad g\left(S_{2} X_{1}, X_{1}\right)=\varepsilon g\left(S_{2} X_{2}, X_{2}\right), \\
& g\left(S_{1} X_{2}, X_{1}\right)=0, \quad g\left(S_{2} X_{1}, X_{2}\right)=g\left(S_{2} X_{2}, X_{1}\right), \\
& -\varepsilon g\left(S_{1} X_{1}, X_{1}\right)+g\left(S_{1} X_{2}, X_{2}\right)+2 g\left(S_{2} X_{1}, X_{2}\right)=0 .
\end{aligned}
$$

Of course (3.3) up to (3.5) remain valid for every orthonormal basis $u=$ $\left\{X_{1}, X_{2}\right\}$ with corresponding transversal basis $\left\{\xi_{1}, \xi_{2}\right\}$.

Similarly, using (3.1), we obtain that

$$
\begin{aligned}
& \left(c^{2}-\varepsilon s^{2}\right)\left(c^{3} C_{111}^{2}+3 c^{2} s C_{112}^{2}+3 c s^{2} C_{122}^{2}+s^{3} C_{222}^{2}\right) \\
& \quad-2 c s\left(c^{3} C_{111}^{1}+3 c^{2} s C_{112}^{1}+3 c s^{2} C_{122}^{1}+s^{3} C_{222}^{1}\right)=0 .
\end{aligned}
$$

Since the above equation has to be valid for all values of $t$, we get that

$$
\begin{array}{lll}
C_{111}^{2}=0, & C_{222}^{2}=0, & C_{222}^{1}=-3 \varepsilon C_{112}^{1}, \\
C_{122}^{2}=2 C_{112}^{1}, & C_{112}^{2}=-2 \varepsilon C_{122}^{1}, & C_{111}^{1}=-3 \varepsilon C_{122}^{1} .
\end{array}
$$


We now restrict our attention to the case that $\sigma$ is either $\sigma_{\mathrm{eq}}$, or $\sigma_{\mathrm{BM}}$, or $\sigma_{\mathrm{WK}}$. By combining the above formulas with the equations characterising these affine transversal planes, it follows in each of the three cases that $C^{1}$ and $C^{2}$ vanish identically. Therefore all three transversal planes coincide and we get the equivalence of (1), (2) and (3). So, in order to complete the proof of the Main Theorem, we may assume that $\sigma=\sigma_{\mathrm{eq}}$ and $C^{1}$ and $C^{2}$ vanish identically. This means that we can use the formulas of Section 8 of [NV]. In particular, we use the orthonormal frame $u=\left\{X_{1}, X_{2}\right\}$ constructed in [NV, p. 162]. For this frame all $\tau$ 's vanish identically, as well as $\nabla_{X_{i}}^{1} X_{j}=0$, for all $i, j \in\{1,2\}$. Equations (8.16) up to (8.19) imply that the shape operators $S_{1}$ and $S_{2}$ satisfy

$$
\begin{array}{ll}
S_{1} X_{1}=\lambda_{1} X_{1}+\lambda_{2} X_{2}, & S_{2} X_{1}=-\varepsilon \lambda_{2} X_{1}+\lambda_{1} X_{2}, \\
S_{1} X_{2}=-\varepsilon \lambda_{2} X_{1}+\lambda_{1} X_{2}, & S_{2} X_{2}=-\varepsilon \lambda_{1} X_{1}-\varepsilon \lambda_{2} X_{2} .
\end{array}
$$

Substituting these equations in (3.3) up to (3.5), we get that $\lambda_{1}=\lambda_{2}=0$. Hence $S_{1}=S_{2}=0$, which since all $\tau$ 's are zero, implies that $\xi_{1}$ and $\xi_{2}$ are constant vector fields in $\mathbb{R}^{4}$. So, since $\nabla_{X_{1}}^{1} X_{2}=\nabla_{X_{2}}^{1} X_{1}(=0)$, there exist coordinates $u$ and $v$ such that

$$
X_{1}=\frac{\partial}{\partial u}, \quad X_{2}=\frac{\partial}{\partial v} .
$$

Therefore, if we denote the immersion of $M^{2}$ into $\mathbb{R}^{4}$ by $x$, we get that

$$
x_{u u}=\xi_{1}, \quad x_{u v}=\xi_{2}, \quad x_{v v}=-\varepsilon \xi_{1} .
$$

Since $\xi_{1}$ and $\xi_{2}$ are both constant vector fields, integration gives that up to an affine transformation $x(u, v)=\left(u, v, \frac{1}{2}\left(u^{2}-\varepsilon v^{2}\right), u v\right)$. If $M^{2}$ is definite the proof is completed. In the indefinite case, after an affine transformation we can rewrite the above example as

$$
x(u, v)=\left(u+v, u-v, \frac{1}{2}(u+v)^{2}, \frac{1}{2}(u-v)^{2}\right) .
$$

A change of variables now completes the proof.

\section{ACKNOWLEDGMENT}

This work was started while the author visited Brown University in April 1993. He would like to thank Professor K. Nomizu for his hospitality and for suggesting this problem.

\section{REFERENCES}

[BM] C. Burstin and W. Mayer, Die Geometrie zweifach ausgedehnter Mannigfaltigkeiten $F_{2}$ im affinen Raum $R_{4}$, Math. Z. 27 (1927), 373-407.

[DMVV] F. Dillen, G. Mys, L. Verstraelen, and L. Vrancken, The affine mean curvature vector for surfaces in $\mathbb{R}^{4}$, Math. Nachr. 166 (1994), 155-165.

[K] W. Klingenberg, Zur affinen Differentialgeometrie, Teil I: Uber p-dimensionale Minimal-flächen und Sphären im n-dimensionalen Raum, Math. Z. 54 (1951), 65-80.

[NV] K. Nomizu and L Vrancken, A new equiaffine theory for surfaces in $\mathbb{R}^{4}$, Internat. J. Math. 4 (1993), 127-165.

[SV] C. Scharlach and L. Vrancken, Affine transversal planes for surfaces in $\mathbb{R}^{4}$, Geometry and Topology of Submanifolds V, World Scientific, Singapore, 1993, pp. 249-253.

Departement Wiskunde, Katholieke Universiteit Leuven, Celestijnenlaan 200 B, B3001, LEUVEN, BeLGiUM

E-mail address: luc.vrancken@ris.kuleuven.ac.be 